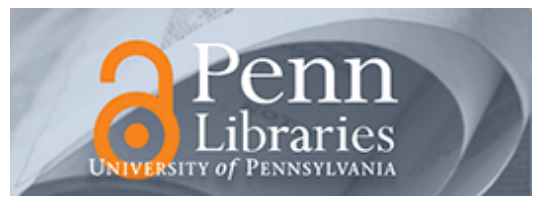

University of Pennsylvania ScholarlyCommons

October 2006

\title{
Tunneling of Electromagnetic Energy through Subwavelength Channels and Bends using $\varepsilon$-Near-Zero Materials
}

Mário Silveirinha

University of Pennsylvania

Nader Engheta

University of Pennsylvania, engheta@seas.upenn.edu

Follow this and additional works at: https://repository.upenn.edu/ese_papers

\section{Recommended Citation}

Mário Silveirinha and Nader Engheta, "Tunneling of Electromagnetic Energy through Subwavelength Channels and Bends using $\varepsilon$-Near-Zero Materials", . October 2006.

Copyright American Physical Society. Reprinted from Physical Review Letters, Volume 97, Issue 15, Article 157403, October 2006, 4 pages.

Publisher URL: http://dx.doi.org/10.1103/PhysRevLett.97.157403

This paper is posted at ScholarlyCommons. https://repository.upenn.edu/ese_papers/213

For more information, please contact repository@pobox.upenn.edu. 


\title{
Tunneling of Electromagnetic Energy through Subwavelength Channels and Bends using $\boldsymbol{\varepsilon}$-Near-Zero Materials
}

\author{
Abstract \\ In this Letter, we demonstrate theoretically that electromagnetic waves can be "squeezed" and tunneled \\ through very narrow channels filled with $\varepsilon$-near-zero (ENZ) materials. We show that the incoming planar \\ wave front is replicated at the output interface, independently of the specific geometry of the channel. A \\ closed analytical formula is derived for the scattering parameters of a particular class of geometries. It is \\ discussed that in some cases the isotropy of the ENZ material may not be an issue. A metamaterial \\ realization of an anisotropic ENZ material is suggested and numerically studied.

\section{Comments} \\ Copyright American Physical Society. Reprinted from Physical Review Letters, Volume 97, Issue 15, Article \\ 157403, October 2006, 4 pages. \\ Publisher URL: http://dx.doi.org/10.1103/PhysRevLett.97.157403
}




\title{
Tunneling of Electromagnetic Energy through Subwavelength Channels and Bends using $\varepsilon$-Near-Zero Materials
}

\author{
Mário Silveirinha* and Nader Engheta ${ }^{\dagger}$ \\ Department of Electrical and Systems Engineering, University of Pennsylvania, Philadelphia, Pennsylvania 19104, USA
}

(Received 23 March 2006; published 10 October 2006)

\begin{abstract}
In this Letter, we demonstrate theoretically that electromagnetic waves can be "squeezed" and tunneled through very narrow channels filled with $\varepsilon$-near-zero (ENZ) materials. We show that the incoming planar wave front is replicated at the output interface, independently of the specific geometry of the channel. A closed analytical formula is derived for the scattering parameters of a particular class of geometries. It is discussed that in some cases the isotropy of the ENZ material may not be an issue. A metamaterial realization of an anisotropic ENZ material is suggested and numerically studied.
\end{abstract}

DOI: 10.1103/PhysRevLett.97.157403

The advent of metamaterials and the flexibility in choosing the value of the permittivity or permeability opened many new possibilities in different fields of physics and engineering. Besides the much celebrated realization of a double negative medium [1] and related prospective applications [2,3], materials with effective $\varepsilon$ and effective $\mu$ or effective refraction index near zero have also become the subject of investigation [4]. Such materials may be found naturally at infrared and optical frequencies, when noble metals, some semiconductors, or plasmonic and polar dielectrics such as $\mathrm{SiC}$ are near their plasma frequency, not to mention plasmas of electron gas. Also, $\varepsilon$-near-zero (ENZ) materials may be properly synthesized as metamaterials at the desired frequency, by embedding suitable inclusions in a host medium. In this Letter we demonstrate that ENZ materials can be used to squeeze electromagnetic energy through narrow subwavelength waveguide channels, and thus to enhance the efficiency of some waveguide devices and reduce the reflection coefficient at a waveguide junction or bend. The physical motivation behind this idea is that since the wavelength of radiation inside the ENZ material is extremely large, the wave must be able to propagate inside the ENZ material with no relevant reflection losses at abrupt bends or junctions. Since ENZ materials have refractive index near zero, the phase variation inside such media is expected to be slow. This suggests that ENZ materials may be used to shape the wave fronts of an electromagnetic wave that propagates through a channel filled with these materials. The radiated wave fronts are expected to be parallel to the geometrical shape of the ENZ material interface. Thus, the efficiency of such waveguide transition must be mainly determined by the ability of the wave penetrating into the ENZ material. Intuitively, one may at first reject this possibility due to the huge impedance contrast between the ENZ material and a regular material. However, as we will prove ahead, if at least one of the physical dimensions of the ENZ material channel is electrically small, the wave may in fact be able to tunnel through the narrow channel. We note that this physical phenomenon is very different from the mechanism de-
PACS numbers: 78.66.Sq, 42.82.Et, 52.40.Db, 52.40.Fd

scribed in [5] to confine light into a nanocavity in the form of surface plasmon polaritons.

To begin with, we first characterize the two-dimensional (2D) scattering of (time harmonic) electromagnetic waves from an ENZ 2D rod with cylindrical symmetry and an arbitrary cross section (apart from being connected). The time variation is assumed to be $\exp (-i \omega t)$, where $\omega$ is the angular frequency. The rod stands in a region invariant to translations along the $z$ direction, and is illuminated by an incoming wave with $z$-oriented magnetic field $\mathbf{H}^{\text {inc }}=$ $\psi^{\text {inc }} \hat{\mathbf{u}}_{z}$, such that $\psi^{\text {inc }}$ is independent of the $z$ coordinate. The total magnetic field is of the form $\mathbf{H}=H_{z} \hat{\mathbf{u}}_{z}$, whereas the electric field is given by $\mathbf{E}=(1 /-i \omega \varepsilon) \bar{\nabla} H_{z} \times \hat{\mathbf{u}}_{z}$. Let us momentarily ignore the effect of losses and suppose that the rod permittivity $\varepsilon$ is identically zero at the operating frequency. Because the electric field must be finite inside the ENZ rod, it is necessary that $\nabla H_{z}$ vanishes and thus the magnetic field must be constant inside the rod, i.e., $H_{z}=H_{z}^{\text {int }}$. On the other hand, in the exterior of the rod the magnetic field is not constant. It is given by the solution of

$$
\boldsymbol{\nabla} \cdot\left(\frac{1}{\varepsilon} \nabla H_{z}\right)+\omega^{2} \mu H_{z}=0
$$

subject to the boundary condition $H_{z}=H_{z}^{\text {int }}$ over the boundary of the ENZ rod, $\partial A$. Owing to the superposition principle, it is possible to write the magnetic field in the exterior of the ENZ rod as $H_{z}=\psi^{\mathrm{PMC}}+H_{z}^{\mathrm{int}} \psi_{1}^{s}$, where $\psi^{\mathrm{PMC}}$ is the total magnetic field when the rod is replaced by a (fictitious) perfect magnetic conductor (PMC) with the same geometry and illuminated with the same plane wave, whereas $\psi_{1}^{s}$ is the exterior solution of (1) subject to the Dirichlet boundary condition $\psi_{1}^{s}=1$ at $\partial A$ and Sommerfeld's radiation conditions at infinity. Note that both $\psi^{\mathrm{PMC}}$ and $\psi_{1}^{s}$ are solutions of exterior mathematical problems. To solve the problem one still needs to determine the unknown constant $H_{z}^{\text {int }}$. To this end, we use Faraday's law to calculate the electromotive force around the contour $\partial A$ : 


$$
\oint_{\partial A} \mathbf{E} \cdot \mathbf{d} \mathbf{l}=+i \omega \mu_{0} \mu_{r, p} H_{z}^{\mathrm{int}} A_{p},
$$

where $\mu_{r, p}$ is the relative permeability of the $\operatorname{rod}$ and $A_{p}$ is its cross-sectional area. Since the tangential component of the electric field is continuous across the boundary of the rod, the expression $H_{z}=\psi^{\mathrm{PMC}}+H_{z}^{\text {int }} \psi_{1}^{s}$ can be used to evaluate the left-hand side of (2). Solving the resulting equation with respect to the unknown $H_{z}^{\text {int }}$, it is found that

$$
H_{z}^{\mathrm{int}}=\frac{-\oint_{\partial A} \frac{1}{\varepsilon_{r}^{\mathrm{ext}}} \frac{\partial \psi^{\mathrm{PMC}}}{\partial n} d l}{\oint_{\partial A} \frac{1}{\varepsilon_{r}^{\mathrm{ext}}} \frac{\partial \psi_{1}^{s}}{\partial n} d l+k_{0}^{2} \mu_{r, p} A_{p}},
$$

where $\partial / \partial n=\hat{\mathbf{n}} \cdot \boldsymbol{\nabla}$ is the normal derivative, $\hat{\mathbf{n}}$ is the outward unit vector normal to $\partial A, k_{0}=\omega \sqrt{\varepsilon_{0} \mu_{0}}$, and $\varepsilon_{r}^{\text {ext }}$ is the relative permittivity of the medium at the outer side of $\partial A$. Next, we will use the above derived results to solve, in closed analytical form, a rather general 2D waveguide problem. The geometry of the structure is as shown in Fig. 1. It consists of two parallel-plate metallic waveguides connected by a channel filled with an ENZ material. It is assumed without loss of generality that the regions delimited by the parallel plates are filled with air and that the metallic walls are perfectly electrical conducting (PEC). The distance between the metallic plates in regions 1 and 2 (see Fig. 1) is, respectively, $a_{1}$ and $a_{2}$. The geometry of the ENZ material section, with the crosssectional area $A_{p}$, is completely arbitrary, except that the input and output faces of this ENZ section are assumed to be perpendicular to the PEC walls in the two parallel-plate waveguide sections. We suppose that the transverse electromagnetic waveguide mode $\psi^{\text {inc }}=H_{z}^{\text {inc }} e^{+i k_{0} x}$ illuminates the ENZ material. Using the theory developed before, it is straightforward to compute the scattering parameters in closed analytical form at a frequency such that the permittivity of the ENZ material vanishes. It is found that $\psi^{\mathrm{PMC}}=H_{z}^{\mathrm{inc}}\left(e^{+i k_{0} x}-e^{-i k_{0} x}\right)$ and $\psi_{1}^{s}=e^{-i k_{0} x}$ in region 1 , and $\psi^{\mathrm{PMC}}=0$ and $\psi_{1}^{s}=e^{+i k_{0} x^{\prime}}$ in region 2 (the origin of the unprimed coordinates is at the first interface,

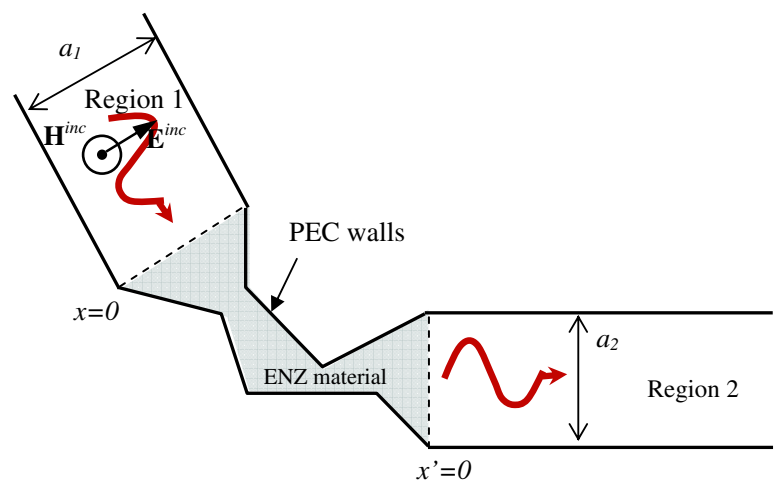

FIG. 1 (color online). Geometry of a generic 2D waveguide structure with an ENZ material section. The waveguide walls in regions 1 and 2 are parallel to the $x$ and $x^{\prime}$ directions, respectively. The interfaces of the ENZ material channel are planar and normal to the waveguide walls. whereas the origin of the primed coordinates is at the second interface, as illustrated in Fig. 1). The magnetic field inside the ENZ region, $H_{z}^{\text {int }}$, can be computed by substituting the previous formulas in (3). Noting that the reflection coefficient for the magnetic field is given by $\rho=$ $-1+H_{z}^{\text {int }} / H_{z}^{\text {inc }}$, we find the following unexpectedly simple result:

$$
\rho=\frac{\left(a_{1}-a_{2}\right)+i k_{0} \mu_{r, p} A_{p}}{\left(a_{1}+a_{2}\right)-i k_{0} \mu_{r, p} A_{p}} .
$$

The above formula is exact and only assumes that the permittivity of the arbitrarily-shaped ENZ material vanishes. For given $a_{1}$ and $a_{2}$ the amplitude of the reflection coefficient satisfies $|\rho| \geq\left|a_{1}-a_{2}\right| /\left(a_{1}+a_{2}\right)$, being the lower bound approached when $k_{0} \mu_{r, p} A_{p}$ is near zero. Thus, in order that the wave can tunnel through the ENZ channel, it is necessary that $a_{1} \approx a_{2}$ and that $k_{0} \mu_{r, p} A_{p} /\left(a_{1}+a_{2}\right) \ll 1$. The condition $a_{1} \approx a_{2}$ can be understood using the energy conservation theorem. In fact, neglecting losses in the ENZ section, the time average power (per unit of length) that enters through the input interface $P_{\text {in }}=\sqrt{\mu_{0} / \varepsilon_{0}}\left|H_{z}^{\text {inc }}\right|^{2}\left(1-|\rho|^{2}\right) a_{1}$ must be the same as the time average power that exits the output interface $P_{\text {out }}=\sqrt{\mu_{0} / \varepsilon_{0}}\left|H_{z}^{\text {inc }}(1+\rho)\right|^{2} a_{2}$ [note that the magnetic field in region 2 is given by $\left.H_{z}=H_{z}^{\text {inc }}(1+\rho) e^{+i k_{0} x^{\prime}}\right]$. But if $\rho$ is near zero this is only possible if $a_{1} \approx a_{2}$. Because of this fact, we will consider that $a_{1}=a_{2} \equiv a$ in the rest of this Letter. Next we discuss the condition $k_{0} \mu_{r, p} A_{p} / 2 a \ll 1$. The first possibility of this condition being achieved is when the relative permeability $\mu_{r, p}$ of the ENZ section is near zero. This is partially expected because when both $\varepsilon$ and $\mu$ are near zero the medium might become matched to the air region [4], and then the wave can tunnel through the zero-index material channel. The second and arguably the most interesting possibility of low reflectance occurs when the cross-sectional area of the ENZ material, $A_{p}$, is electrically small, or more generally when at least one of the physical dimensions of the ENZ channel is electrically small $\left(k_{0} A_{p} / 2 a \ll 1\right)$. Note that for $a$ fixed (and possibly electrically large) it is always possible to design the ENZ channel in such a way that $k_{0} A_{p} / 2 a$ can be arbitrarily small by decreasing the channel width. The electrodynamics of a lossless ENZ medium is thus very peculiar. In effect, as the ENZ channel becomes increasingly narrower, the reflectance decreases even more. Because of the conservation of energy and of Poynting's theorem, the amplitude of the electric field inside the ENZ material must also increase when the width of the channel is decreased. So, in practice, the minimal channel width will be limited by the dielectric or wall losses and possible dielectric breakdown. Although the geometry of this problem is intrinsically two dimensional, the results can also be extended to an important class of 3D-waveguide problems.

In order to illustrate some of the possibilities suggested by our analysis, we consider the geometry shown in Fig. 2. 


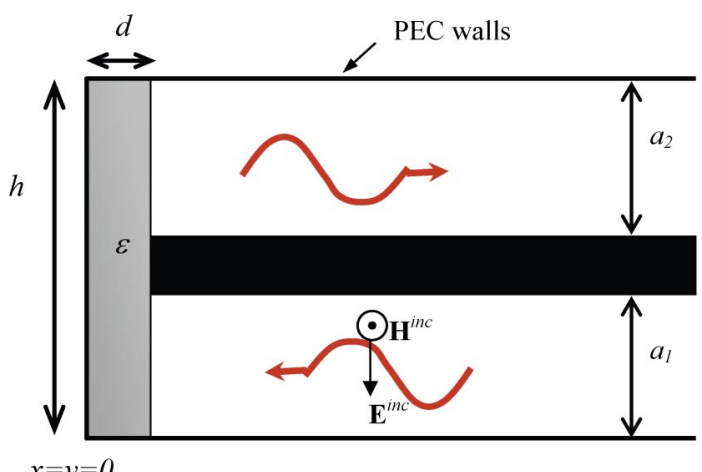

FIG. 2 (color online). Geometry of a 2D parallel-plate waveguide, with a $180^{\circ}$ bend that is filled with an ENZ material.

The structure consists of two 2D parallel-plate waveguides, parallel to each other, and forming a $180^{\circ}$ bend. First, we consider the case in which the distance between the two waveguides is infinitesimally small, $h=a_{1}+a_{2}=2 a$, and the thickness of the bend is also small, e.g., $d=$ $0.1 a$. Let us suppose that the bend is filled with a (nonmagnetic) material with permittivity following a Drudetype dispersion model $\varepsilon=\varepsilon_{0}\left(1-\frac{\omega_{p}^{2}}{\omega(\omega+i \Gamma)}\right)$, where $\omega_{p}$ is the plasma frequency, and $\Gamma$ is the collision frequency $(\mathrm{rad} / \mathrm{s})$. In the first example, we suppose that $\omega_{p} a / c=$ 2.0 ( $c$ is the speed of light in vacuum). The amplitude of the reflection coefficient is depicted in Fig. 3 as a function of normalized frequency $\omega a / c$. The curves were calculated using the commercial simulator CST Microwave Studio ${ }^{\text {TM }}$ [6]. As seen in Fig. 3, several transmission windows are revealed when $\Gamma / \omega_{p}=0$ (curve $a$ ) and when $\Gamma / \omega_{p}=$ 0.05 (curve $b$ ). When the ENZ material is removed (curve $c$ ) transmission is not possible, except in the static limit. As expected, around $\omega a / c=2.0$ the reflection coefficient has a dip, even when losses are considered (curve $b$ ) [note that at $\omega=\omega_{p}$, we have $\operatorname{Re}(\varepsilon) \approx 0$ and $\left.\varepsilon / \varepsilon_{0} \approx+i \Gamma / \omega_{p}\right]$. Equation (4) predicts that for this geometry $|\rho|=0.2$ when $\varepsilon=0$. This value is consistent with the full wave numerical results. Note that in curve $a$ the null of $\rho$ occurs for some frequency slightly above $\omega_{p}$. Also, it is seen that for $\omega<\omega_{p}$, where $\operatorname{Re}\{\varepsilon\}<0$, the behavior of $|\rho|$ is highly oscillatory when losses are vanishingly small (especially for $\operatorname{Re}\{\varepsilon\}<-1$ ). It can be proved that this oscillatory behavior occurs due to very strong singularities and resonances of the fields near the edge of the metallic plate. Qualitatively, these resonances are closely related to the well-known "quasistatic" resonances [7] that arise in objects with negative permittivity embedded in a positive permittivity background. As shown in curve $b$, when losses are considered these oscillations tend to disappear. However, the amplitude of the reflection coefficient near the plasma frequency [where $\operatorname{Re}(\varepsilon) \approx 0$ ] is small and to a large extent unaffected by the losses. In fact, the dip in the reflection characteristic at $\omega=\omega_{p}$ is not related to any resonance phenomenon intrinsic to the ge-

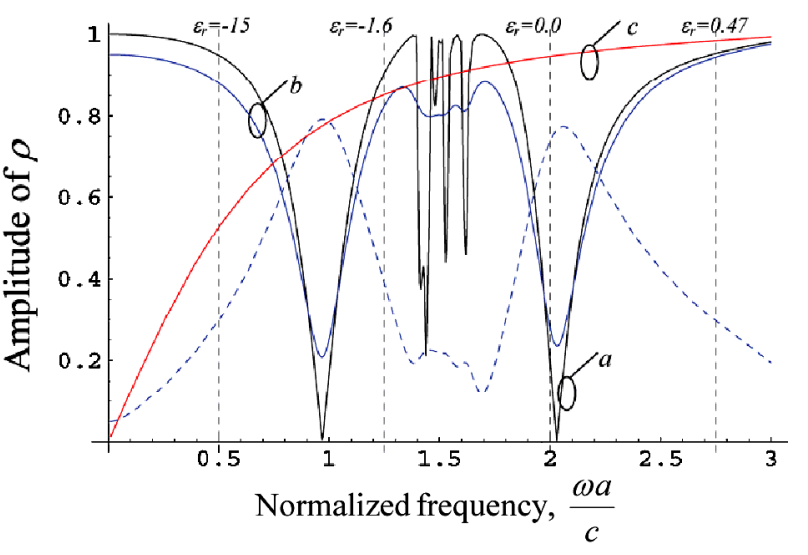

FIG. 3 (color online). Reflection coefficient magnitude as a function of the normalized frequency. Curve $a$ : bend filled with lossless ENZ material. Curve $b$ : bend filled with lossy ENZ material, $\Gamma / \omega_{p}=0.05$. Curve $c$ : unfilled bend. The dashed vertical lines indicate the value of $\varepsilon_{r}$ at the corresponding frequency. The dashed curve represents the transmission characteristic corresponding to curve $b$, clearly highlighting the tunneling of energy through this channel.

ometry and shape of the structure, but instead is due to the peculiar electromagnetic properties of ENZ media. In Fig. 4 the contour plot of the amplitude of the electric field [in panel (a)] and the real part of the Poynting vector lines [in panel (b)] are shown at $\omega=\omega_{p}$. As expected, the amplitude of the electric field is larger inside the ENZ material, and the flow of real part of the Poynting vector shows how the power "squeezes" through the narrow channel in order to tunnel through it. Using the modematching technique, the fields can be calculated in closed analytical form in the $\varepsilon=0$ limit. To a first-order approximation the electric field inside the bend is $\frac{\mathbf{E}}{\eta_{0} H_{z}^{\text {inc }}} \approx$ $\frac{(y-2 a)}{d} \hat{\mathbf{u}}_{x}+\frac{x}{d}(1+\rho) \hat{\mathbf{u}}_{y}$ for $y \geq a$ and $\frac{\mathbf{E}}{\eta_{0} H_{z}^{\mathrm{mic}}} \approx-\frac{y}{d} \hat{\mathbf{u}}_{x}-$ $\frac{x}{d}(1-\rho) \hat{\mathbf{u}}_{y}$ for $y \leq a$, where $\eta_{0}=\sqrt{\mu_{0} / \varepsilon_{0}}$, and the $x$ and $y$ coordinates are along the parallel plates of the waveguides and normal to it, respectively. The formula is particularly accurate when $d \ll a$ and $y \neq a$. It shows that the
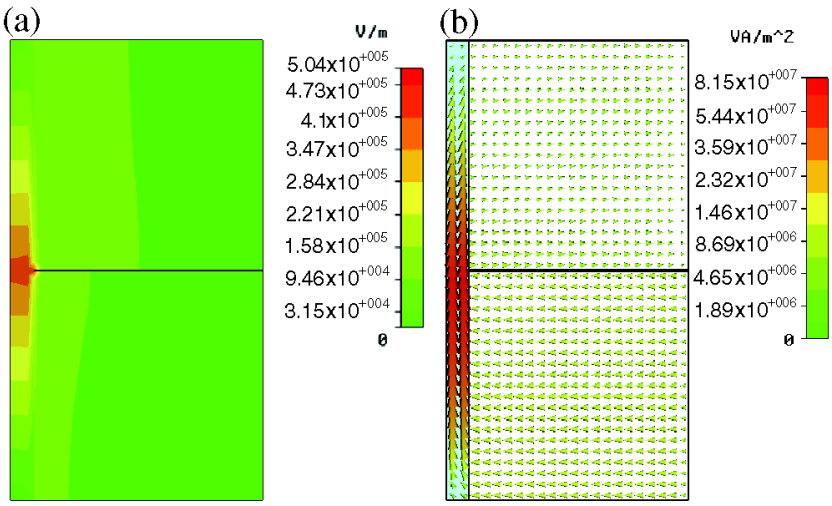

FIG. 4 (color online). (a) Contour plot of the amplitude of the electric field. (b) Real part of the Poynting vector lines. 
electric field grows as $1 / d$ as the thickness of the ENZ section decreases.

At infrared and optical frequencies, ENZ materials may be available either naturally or by mixing plasmonic materials with conventional dielectrics. In these regions of the electromagnetic spectrum PEC materials may not be readily available because metals lose their usual conducting properties. Nevertheless, Ag, Al, Au, and other metals have a real part of the permittivity that can be very negative and thus to a first approximation may work effectively as "good" conducting materials. On the other hand, at the microwave and millimeter-wave frequencies, where good conductors are readily accessible, ENZ materials may not readily be available. In this case, a practical implementation of the described structure requires in principle the use of metamaterials. It is known [8] that a 3D-array of connected metallic wires behaves to a first approximation as an isotropic plasma. However, a 3D wire mesh is difficult to characterize numerically, and above all it may be challenging to fabricate. Since anisotropic metamaterials are in general relatively easier to assemble, it is interesting to study if the "squeezing" and tunneling effect revealed above can also be obtained with ENZ anisotropic materials. Our theoretical study similar to the one presented in the first part of this Letter shows that for the geometry of Fig. 2 the effect is still revealed if $\varepsilon_{x x}=0$ (we consider that the principal axes of the permittivity tensor are directed along the coordinate axes). More specifically, for this case the reflection coefficient is given by $\rho=$ $\frac{\left(a_{1}-a_{2}\right) \varepsilon_{y y, r} k_{0}+i h k_{x} \tan \left(k_{x} d\right)}{\left(a_{1}+a_{2}\right) \varepsilon_{y y, r} k_{0}-i h k_{x} \tan \left(k_{x} d\right)}$, where $k_{x}=k_{0} \sqrt{\varepsilon_{y y, r} \mu_{r, p}}$, and $\varepsilon_{y y, r}$ is the relative permittivity along the $y$ direction. Assuming that $a_{1}=a_{2} \equiv a$, the reflection coefficient can be made arbitrarily small by decreasing the thickness $d$ of the ENZ anisotropic material. It is known that a square lattice of thin metallic wires directed along the $x$ direction behaves effectively as an anisotropic ENZ material with $\varepsilon_{x x}=0$ at a certain plasma frequency. Based on this idea a ENZ material was designed with dimensions $h=3 a$ and $d=0.1 a$. The wires are embedded in a dielectric host with relative permittivity $\varepsilon_{r \text {,host }}$. The length and radius of the wires are, respectively, $d$ and $r_{w}$. The wires are centered at $(0,0.5+n, m) s_{w}, n=0,1,2, \ldots, 5, m=0, \pm 1, \pm 2, \ldots$, as illustrated in the inset of Fig. 5 (each cell contains 6 wires; the spacing between the wires is $s_{w}=0.5 a$ ). Assuming that $r_{w}=0.01 s_{w}$, it is known that the plasma frequency of the associated wire medium (formed by infinitely long wires) is such that $\omega_{p} \sqrt{\varepsilon_{r, \text { host }}} s_{w} / c=1.37$. Thus, choosing $\varepsilon_{r \text {,host }}=4.0$, the plasma frequency is such that $\omega_{p} a / c=1.37$. The reflection characteristic was computed using a full wave electromagnetic simulator [6]. In Fig. 5, the amplitude $|\rho|$ is shown as function of frequency for (curve $a$ ) metamaterial bend using the wire inclusions mentioned above, (curve $b$ ) ideal anisotropic material with $\varepsilon_{x x}$ following the Drude model and $\varepsilon_{y y, r}=$ $\varepsilon_{r, \text { host }}$, and (curve $c$ ) unfilled bend. It is seen that around the

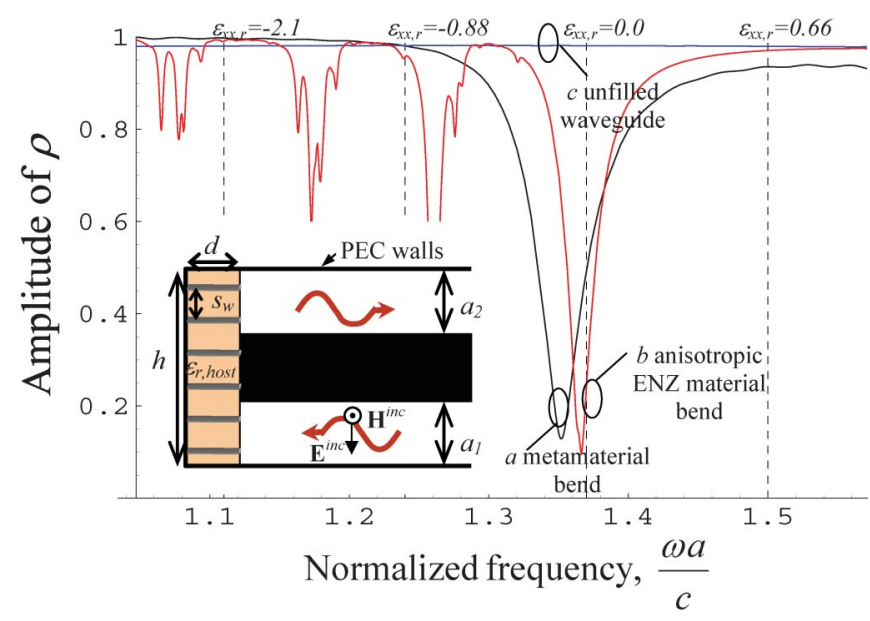

FIG. 5 (color online). Reflection coefficient as a function of normalized frequency. Curve $a$ : bend filled with wire-medium metamaterial. Curve $b$ : bend filled with anisotropic ENZ material. Curve $c$ : unfilled bend. The dashed vertical lines indicate the value of $\varepsilon_{x x, r}$ at the corresponding frequency. The inset shows the geometry of the parallel-plate waveguide with a bend filled with anisotropic metamaterial using $x$-oriented wires.

design plasma frequency the reflection characteristic has a dip. The dip is slightly shifted in frequency perhaps due to the fact that the number of wires per unit cell is relatively small or the interface effects. The spatial dispersion of the wire medium [8] may also play a role here.

In conclusion, we have shown that tunneling and squeezing electromagnetic energy through subwavelength narrow channels and waveguide bends are possible using ENZ materials, and that ENZ-metamaterial bends can play interesting roles in reducing the reflectivity at certain waveguide bends with potential applications in miniaturization of optical energy transports and interconnects.

This work is supported by U.S. AFOSR Grant No. FA9550-05-1-0442. M. S. has been partially supported by Fundação para a Ciência e Tecnologia.

*On sabbatical leave from Instituto de Telecomunicações, Universidade de Coimbra, Portugal.

${ }^{\dagger}$ To whom correspondence should be addressed. Electronic address: engheta@ee.upenn.edu

[1] D. R. Smith, W. J. Padilla, D. C. Vier, S. C. Nemat-Nasser, and S. Schultz, Phys. Rev. Lett. 84, 4184 (2000).

[2] J. B. Pendry, Phys. Rev. Lett. 85, 3966 (2000).

[3] N. Engheta, IEEE AWPL 1, 10 (2002).

[4] R. W. Ziolkowski, Phys. Rev. E 70, 046608 (2004).

[5] Hideki T. Miyazaki and Yoichi Kurokawa, Phys. Rev. Lett. 96, 097401 (2006).

[6] CST Microwave Studio ${ }^{\mathrm{TM}}$ 5.0, CST of America, Inc., www.cst.com.

[7] D. R. Fredkin and I. D. Mayergoyz, Phys. Rev. Lett. 91, 253902 (2003).

[8] M. Silveirinha and C.A. Fernandes, IEEE Trans. Microwave Theory Tech. 53, 1418 (2005). 